\title{
Asociaciones fitoplanctónicas y su periodicidad en un lago marcadamente estratificado
}

\author{
S. M. Morata, A. Camacho, M. R. Miracle y E. Vicente \\ Departamento de Microbiología y Ecología e Instituto Cavanilles de Biodiversidad y Biología Evolutiva, \\ Universitat de València. E-46100 Burjassot, España.
}

\section{RESUMEN}

La Laguna del Tejo es un lago cárstico cerrado, que durante el periodo estudiado (1997-1998) presentaba una situación de oligotrofia en su epilimnion pero no en sus aguas profundas. El alto grado de estratificación estival produce una organización vertical del plancton, de tal manera que en el epilimnion no se produce el reciclaje completo de los nutrientes, sino que se acumulan por sedimentación en el hipolimnion, anóxico en su parte profunda y en cuya interfase óxico-anóxica, a la que llega la luz, se sitúa el máximo profundo de clorofila formado fundamentalmente por picocianobacterias. Respecto del nanofitoplancton, la sucesión en superficie, regida por la estabilidad del agua y el agotamiento de nutrientes, viene definida al igual que en otros lagos por la secuencia: Cyclotella wuethrichiana - Peridinium spp. - Ceratium hirundinella. Las dos primeras especies se hacen metalimnéticas a medida que avanza la sucesión. Por otra parte las criptofíceas, abundantes también en la mezcla invernal, se sitúan durante la estratificación preferentemente en el hipolimnion próximas a la interfase óxico-anóxica, estableciéndose por encima de ellas una capa de crisófitos.

Singular en este lago es la regresión de la sucesión ecológica definida por la secuencia: dinoflageladas - desmidiáceas, que se da a mediados-finales del periodo de estratificación, cuando las perturbaciones físicas actúan profundizando la termoclina. Esta regresión se manifiesta en este lago por la proliferación de Cosmarium spp. y presenta una acusada variación interanual. En 1998 se produjo un intenso crecimiento de Cosmarium cf. bioculatum en el metalimnion, con abundancias superiores en más de un orden de magnitud a las demás algas, hecho que se relaciona con variaciones notables en las condiciones de la laguna respecto al mismo periodo del año anterior, en el que no se produjo un crecimiento explosivo. El análisis de componentes principales realizado pone de manifiesto las variaciones mencionadas, dando una primera componente que contrapone el inicio y el final de la estratificación (Peridinium spp.-Cosmarium spp.), así como las variaciones en el perfil vertical, y una segunda componente que destaca las características diferenciales de mediados de verano-otoño de 1998, asociadas sobre todo al crecimiento masivo de Cosmarium bioculatum y C. cf. bioculatum, contrastándolo especialmente con las condiciones del mismo periodo en 1997. Este factor principal también diferencia la posición en diferentes profundidades de las especies y tiene una fuerte correlación positiva con Bitrichia sp., crisófito favorecido en la estratificación de 1998. Los resultados del análisis también indican que existe una gran similitud entre la comunidad fitoplanctónica del periodo de mezcla y la de las capas más profundas del hipolimnion estival. Diversidad y abundancia muestran correlaciones negativas y se observa una baja diversidad a finales de verano-otoño del año 1998 contrastando con la diversidad mucho más alta del año anterior.

Palabras clave: Lagos cársticos, nanofitoplancton, picoplancton, distribución vertical, máximo profundo de clorofila, limitación por nutrientes, sucesión ecológica, Cosmarium, Cyclotella.

\begin{abstract}
Lake El Tejo is a closed karstic lake. During the studied period (1997-1998) there was a relatively low availability of inorganic nutrients, driving to oligotrophic conditions in the surface waters but not in deeper layers. The steep summer stratification of its waters produced a vertical organization of the plankton, with an epiliminion where nutrient recycling was not completed and a hypolimnion that became anoxic in its deeper part. At the oxic-anoxic interface, where light still arrives, a deep chlorophyll maximum was found, mostly formed by picocyanobacteria. Regarding nanophytoplankton, the succession in surface waters was similar to that of other lakes, governed by the stability of the water and the epilimnetic exhaustion of nutrients, with the sequence: Cyclotella wuethrichiana - Peridinium spp. - Ceratium hirundinella. The first two species became metalimnetic as the succession advanced. On the other hand, Cryptophyceae, which were also abundant during winter mixis, during stratification were located close to the oxic-anoxic interface, with Chrysophyceae in the immediate layers above the Cryptophyceae.
\end{abstract}


What was remarkable about this lake during the study period was the regression in the ecological succession: DinophyceaeDesmids, occurring in the second half of the stratification period, when the thermocline became deeper. This regression was characterized by the occurrence of a bloom of Cosmarium ssp. and showed marked interannual variation. Thus, in 1998, an intense metalimnetic bloom of Cosmarium cf. bioculatum occurred, with abundances of an order of magnitude higher than the other algae. This was probably related to the large changes in lacustrine conditions compared to the same period of the previous year, when such explosive growth did not occur. A principal component analysis of the nanophytoplankton data gave a first component which represented change between beginning and end of the stratification period (Peridinium spp. Cosmarium spp.), as well as associated differences in the vertical profile. The second component highlighted the differential characteristics of the second half of the stratification period of 1998, as compared to the other years, i.e. the massive growth of C. bioculatum and C. cf. bioculatum in contrast with the same period of 1997. This component also underlines the depth of maxima of different species. It, showed a strong positive correlation with the maximum of Bitrichia sp. a chrysophycean algae which appeared during the stratification period of 1998. The PCA analysis also showed the high similarity between the phytoplankton community during the mixing period and that in deeper waters during summer. Diversity and abundance were inversely correlated. Low diversity in the second half of the stratification period of 1998 was in stark contrast with the much higher diversity of the same period of the previous year.

Keywords: Karstic lakes, nanophytoplankton, picoplankton, vertical distribution, deep chlorophyll maxima, nutrient limitation, ecological succession, Cosmarium, Cyclotella.

\section{INTRODUCCIÓN}

La incidencia de la estabilidad generada durante la estratificación térmica sobre los organismos planctónicos ha sido descrita en varios trabajos (Reynolds, 1992; Miracle et al., 1998), y en último término implica una estrecha vinculación entre las relaciones tróficas y los factores ambientales. Los lagos cársticos de profundidades relativas muy altas, muestran marcados gradientes verticales de temperatura, con un notable máximo metalimnético de oxígeno resultante de la fotosíntesis algal, generándose en el hipolimnion una oxiclina, en cuya parte inferior se mantienen también altas densidades fitoplanctónicas. Los estudios en lagos de estas características aportan pues una valiosa información sobre las comunidades fitoplanctónicas que se pueden organizar en el eje vertical luznutrientes de la columna de agua. Además, dada la escasez de estudios sobre productores primarios en lagos de origen cárstico (Planas, 1990), no se han llegado a establecer claramente las asociaciones de especies que se dan en los diferentes estratos. Si bien existen algunos estudios del fitoplancton en lagos cársticos en la Península Ibérica, la Laguna de la Cruz (Rojo y Miracle, 1987; Dasí y Miracle, 1991), el Lago de Banyoles (Planas, 1973), la Laguna del Vilà
(García de Emiliani, 1973), el Lago de Montcortés (Camps et al., 1976) y el Lago Grande de Estanya (Ávila et al., 1984), las poblaciones fitoplanctónicas son bastante diferentes en cuanto a su composición específica y tipología, siendo necesarios más estudios en estos u otros lagos cársticos para determinar las principales relaciones de las comunidades fitoplanctónicas con los procesos de estratificación de las aguas, tan acusados en estos lagos.

Con objeto de determinar la distribución vertical de las poblaciones algales (microplancton y nanoplancton fotosintético) y su relación con los gradientes de temperatura y oxígeno durante su evolución estacional en un lago de las antedichas características, se realizó un estudio en una pequeña laguna cárstica durante los años 1997-1998, que estaba enmarcado en un proyecto más amplio enfocado al análisis de la transferencia de nutrientes entre los distintos componentes de la red trófica.

\section{MATERIAL Y MÉTODOS}

\section{Lugar de estudio}

La Laguna del Tejo es la más profunda del conjunto lagunar de las Torcas de Cañada del 
Hoyo $\left(1^{\circ} 52^{\prime} 37^{\prime \prime} \mathrm{W} 39^{\circ} 59^{\prime} 20^{\prime \prime} \mathrm{N}\right)$, situadas a unos $25 \mathrm{~km}$ de Cuenca (España) y a unos $1000 \mathrm{~m}$ de altitud sobre el nivel del mar. Se trata de una dolina circular cerrada, formada por disolución y colapso de un sustrato dolomítico, que al estar condicionada por una falla lístrica, el desplazamiento de la formación "margas de Chera" (Cenomanense) que subyace al paquete de dolomias, impermeabiliza el área de descarga del vaso y retiene el agua de un acuífero local (Santisteban, comunicación personal). De este modo el volumen de agua en la cubeta está en equilibrio con el nivel del acuífero que la circunda, por lo que las entradas de agua subterránea compensan hasta cierto punto las pérdidas por evaporación. Durante el periodo de estudio, la Laguna del Tejo presentaba una superficie aproximada de 1.6 ha y una profundidad máxima fluctuando alrededor de los $28 \mathrm{~m}$.

\section{Muestreo y métodos analíticos}

Los muestreos se realizaron mensualmente a partir de septiembre de 1997 hasta diciembre de 1998 en dos puntos próximos al centro de la Laguna del Tejo. Se determinaron in situ a lo largo del perfil vertical las siguientes variables: temperatura, conductividad, concentración de oxígeno disuelto y $\mathrm{pH}$ utilizando las correspondientes sondas de medida y la penetración de la luz a partir del disco de Secchi. En base a la distribución de estas variables se eligieron las distintas profundidades de muestreo que se realizó con una botella hidrográfica de Ruttner, recogiéndose para los recuentos de fitoplancton muestras de agua generalmente a $0.5,3,8,11,14,16,19,21$ y $24 \mathrm{~m}$. En el año 1998 se recogieron adicionalmente a $18 \mathrm{~m}$. Durante el periodo de diciembre a febrero se suprimieron algunas de las profundidades intermedias (11 y $14 \mathrm{~m}$, en general) y las muestras de $24 \mathrm{~m}$ cuando estaban en aguas anóxicas durante la estratificación no se han considerado en los análisis estadísticos del fitoplancton. En el laboratorio se determinaron los nutrientes inorgánicos $(\mathrm{N}, \mathrm{P}, \mathrm{Si})$ siguiendo la metodología descrita por APHA-AWWAWEF (1992) y Golterman et al. (1978). La cuantificación de las clorofilas se realizó aplicando las ecuaciones propuestas por Jeffrey \& Humphrey (1975) a las absorbancias del extracto pigmentario en acetona-dimetilsulfóxido (Shoaf \& Lium, 1976).

\section{Recuentos de fitoplancton}

Las muestras de agua, recogidas en botellas de cristal topacio de $250 \mathrm{ml}$ y fijadas in situ con una solución de lugol, se procesaron en el laboratorio siguiendo el método de Utermöhl (1958), realizándose el recuento por observación en DIC con un microscopio invertido Zeiss a 1000X y 400X. La identificación de las especies nanofitoplanctónicas se ha basado en las claves taxonómicas de los siguientes autores: Bacillariophyceae en Germain (1981) y Krammer \& Large-Bertalot (1986, 1988, 1991), Chlorophyceae en Förster (1982) y Komárek \& Fott (1983), Cryptophyceae en HuberPestalozzi (1968) y Anton \& Duthie (1981), Chrysophyceae en Starmach (1985), Euglenophyceae en Huber-Pestalozzi (1955), Dinophyceae en Popovský \& Pfiester (1990) y Cyanophyceae en Anagnostidis \& Komárek (1988). Se observó una gran cantidad de células picoplanctónicas (Camacho et al., 2003) cuyo recuento se realizó por técnicas de microscopía de fluorescencia.

Las abundancias y diversidades, calculadas según el índice de Shannon-Wiener (Shannon \& Weaver, 1963), se analizaron en tres intervalos de profundidad coherentes con las capas generadas durante la época de estratificación: (1) epilimnion $(0.5$ y $3 \mathrm{~m}$ en junio y julio, agregando la de $8 \mathrm{~m}$ en agosto, la de $11 \mathrm{~m}$ en septiembre 1998 y mediados de octubre de 1997, pero no en septiembre-primeros de octubre de 1997 y en noviembre de los dos años la de $14 \mathrm{~m}$ ), (2) metalimnion-hipolimnion (siempre hasta los $18 \mathrm{~m}$ ) y (3) parte final de la oxiclina (siempre desde los $19 \mathrm{~m}$ ). Durante la mezcla vertical (diciembre -mayo) se agruparon de 0-10 m, 11-18 $\mathrm{m}$ y $19-24 \mathrm{~m}$. 

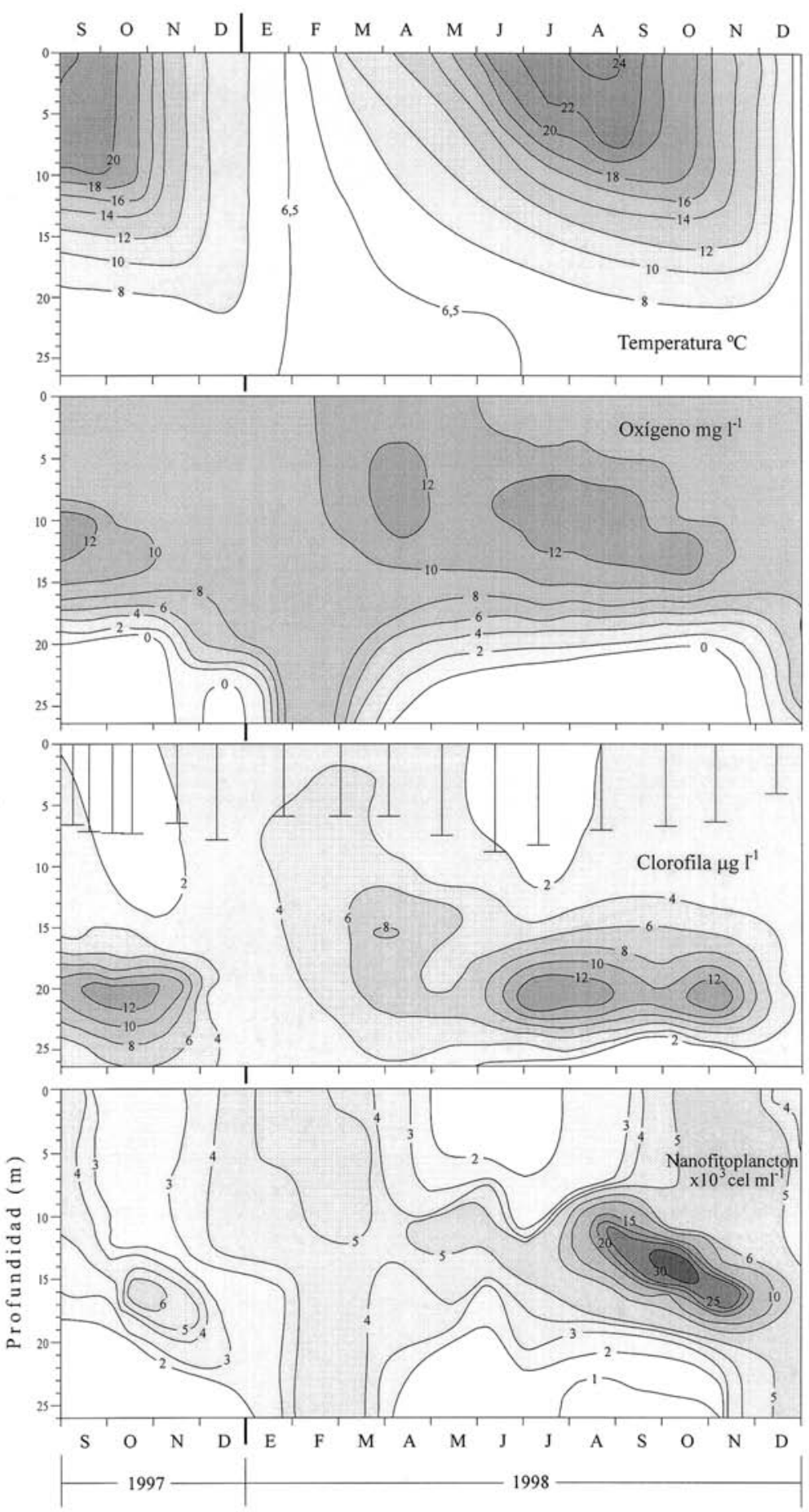

Figura 1. Isolíneas de temperatura, oxígeno, clorofila $a$ y densidad total del nanofitoplancton desde septiembre 1997 hasta diciembre de 1998. En la gráfica de clorofila $a$ se incluye la profundidad del disco de Secchi (m) para cada muestreo. Time-depth isopleths of temperature, oxygen, chlorophyll-a and total nanophytoplankton density from September 1997 to December 1998. In the graph of chlorophyll-a, the Secchi disk depth $(m)$ is shown for each sampling occasion. 
Se aplicó un Análisis de Componentes Principales (PCA) para los datos referentes a las 18 especies principales de la laguna, previamente normalizados $(\ln (\mathrm{x}+1)$, siendo $x$ el número de individuos por unidad de volumen de cada especie) mediante el programa SPSS 10.0.

\section{RESULTADOS}

\section{Características limnológicas}

En el año 1998, la Laguna del Tejo presentó un largo periodo de estratificación térmica desde finales de primavera hasta mediados de otoño con máximos de $24^{\circ} \mathrm{C}$ en superficie en agosto $\mathrm{y}$ un periodo de mezcla vertical completa en invierno, siendo los mínimos de temperatura registrados $6.3^{\circ} \mathrm{C}$ en febrero y permaneciendo el agua profunda con valores próximos a ese mínimo (Fig. 1). A lo largo del periodo de estratificación los máximos de oxígeno se localizaron en la parte superior de la termoclina, variando su posición desde $5 \mathrm{~m}$ a principios de junio a $12 \mathrm{~m}$ a finales de septiembre (Fig. 1). En el hipolimnion profundo se estableció una zona anóxica, la interfase óxicoanóxica situada a los $22.5 \mathrm{~m}$ de profundidad a principios de mayo ascendió hasta $\operatorname{los} 20 \mathrm{~m} \mathrm{a}$ finales de septiembre. En el año anterior las condiciones del final de la estratificación y de la mezcla otoñal fueron algo diferentes: el 30 de septiembre de 1997 la temperatura epilimnética fue un par de grados más alta, empezando la termoclina y la interfase óxico-anóxica ambas medio metro más arriba que el mismo día de 1998. Por otro lado el máximo metalimnético de oxígeno fue mucho menor y restringido a un intervalo de profundidades también menor. Los máximos metalimnéticos de oxígeno se correspondieron con zonas de clorofila de concentraciones intermedias y de inicio de máximos de densidad nanofitoplanctónica (Fig. 1), por lo que se relacionan con la actividad fotosintética y acumulación de oxígeno, a consecuencia de la restricción de la difusión, en aguas fuertemente estratificadas.
La concentración de clorofila $a$ (Fig. 1) fue relativamente baja y homogénea en toda la columna de agua durante la época invernal y presentó un marcado máximo primaveral en la mayor parte del perfil vertical. Durante los periodos de estratificación, en cambio, los valores fueron mínimos en el epilimnion pero se localizó un máximo profundo en torno a los $20 \mathrm{~m}$ de profundidad debido mayoritariamente a una densa población de picocianobacterias, cuyo estudio no se incluye en el presente trabajo. Por esto la distribución de clorofila no coincidía con la densidad del nanofitoplancton (Fig. 1), presentando éste los máximos algo por encima (14-16 m) de los de clorofila. A lo largo del estudio la conductividad se mantuvo en torno a los 490-520 $\mu \mathrm{S} / \mathrm{cm}$ en la zona bien oxigenada del perfil vertical, incrementándose ligeramente en la zona anóxica. Los valores de $\mathrm{pH}$ se encontraban en el rango de 8.5-9.1 en la zona óxica. La concentración de compuestos inorgánicos de fósforo y nitrógeno fue muy baja; los valores de fósforo reactivo soluble (SRP) oscilaron entre valores indetectables y 0.20 $\mu \mathrm{M}$ y el nitrógeno inorgánico disuelto (DIN) varió de forma importante tanto en el tiempo como en el perfil vertical, oscilando entre valores indetectables hasta $1 \mu \mathrm{M}$ en invierno. En la primavera presentó los valores más altos alcanzándose los 10-15 $\mu \mathrm{M}$ en la zona oxigenada de la columna de agua. A partir de julio, cuando la estratificación estaba bien establecida el DIN volvió a disminuir a valores menores de $2 \mu \mathrm{M}$, sin considerar las aguas anóxicas y permanecieron bajos hasta el final de la estratificación, aunque ligeramente superiores a los del año anterior. Sin embargo se produjo una mezcla otoñal mucho más temprana y completa en 1998 que proporcionó mayores concentraciones de DIN en esta época que en el año anterior. Respecto a los silicatos, se detectaron en mínima cantidad a lo largo de la estratificación y aumentaron al comienzo de los periodos de mezcla, localizándose las mayores concentraciones en aguas profundas. 
Tabla 1. Relación de tamaños observados (L: longitud máxima; A: anchura máxima) y características destacables de algunas de las principales especies nanofitoplanctónicas. Observed sizes (L: maximum length; A: maximum width) and taxonomic features from main nanophytoplanktonic species.

\begin{tabular}{lcl}
\hline Especies & L $\mathbf{x}(\mu \mathbf{m})$ & Características destacables \\
\hline $\begin{array}{l}\text { Diatomeas } \\
\text { Cyclotella wuethrichiana }\end{array}$ & $\begin{array}{c}4-7 \\
\text { (vista valvar) }\end{array}$ & $\begin{array}{l}\text { Finas estrias radiales marginales, una de las cuales se alarga hasta } \\
\text { un proceso reforzado hacia la zona central de la valva. }\end{array}$ \\
$\begin{array}{l}\text { Synedra acus } \\
\text { (vista valvar) }\end{array}$ & $\begin{array}{l}\text { Finas estrias transversales paralelas y alternas a cada lado del } \\
\text { esternón, ausentes en el centro. Valvas líneo-lanceoladas afiladas } \\
\text { hacia los extremos y con polos ligeramente abultados y redondeados. }\end{array}$
\end{tabular}

Dinoflagelados

Peridinium umbonatum 17-25 x 18-24

Clorofíceas

Cosmarium cf. bioculatum $\quad 8-10 \times 7-9$

Chlorella cf. vulgaris $\quad 5-8$

Crisofíceas

Bitrichia sp.
5-8* Célula en cápsula hialina con 2 largos y robustos apéndices en forma 15-30** cónica, poco o nada curvados y de la misma longitud. Morfología y tamaño similar a B.phaseolus (Fott/Bourrelly). No se observó opérculo.

* (célula)

** (longitud apéndice)

\section{Distribución vertical y evolución estacional del nanofitoplancton}

Las especies nanofitoplanctónicas encontradas son propias de las lagunas cársticas de la Península Ibérica. En la Tabla 1 se muestran ciertas características relevantes de algunas de las especies principales encontradas en la Laguna del Tejo, especialmente las de difícil identificación y las menos citadas como dominantes en otros trabajos sobre dichas lagunas. La distribución espacio-temporal de las principales especies nanofitoplanctónicas presentes en la Laguna del Tejo permitió establecer grupos algales dependiendo de la posición que ocuparon en la columna de agua (Fig. 2). A conti- nuación se describen las especies agrupadas según su ubicación en las capas de agua en la que cada especie se desarrolló más habitualmente y/o de modo más intenso a lo largo del periodo estudiado.

\section{a) Especies de presencia constante.}

Cyclotella wuethrichiana (Druart \& Straub) fue la especie mayoritaria durante todo el periodo de estudio, siendo además la especie más frecuente prácticamente en toda la columna de agua y la que alcanzó densidades mayores, a excepción de Cosmarium cf. bioculatum en su máximo puntual de crecimiento. La mayor densidad de C. wuethrichiana se detectó durante la 
Fitoplancton de un lago estratificado
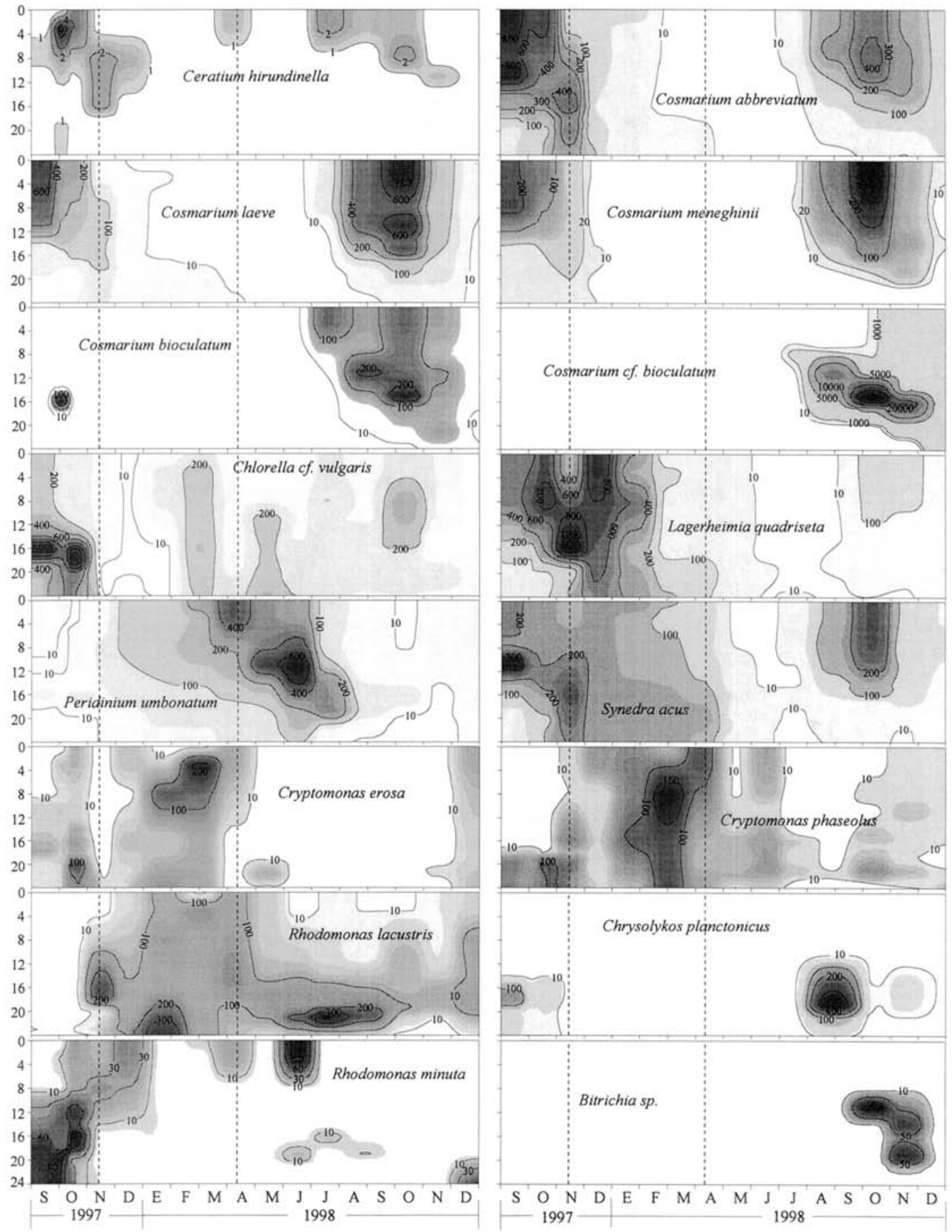

Figura 2. Isolíneas de la densidad (cel ml-1) de las principales especies del nanofitoplancton durante el periodo de muestreo (meses, en abscisas) y en el perfil vertical (profundidad $\mathrm{m}$, en ordenadas). Las líneas discontinuas enmarcan el periodo de mezcla vertical de las aguas. Time-depth distribution of main nanophytoplanktonic species. Density isopleths in cel $\mathrm{ml}^{-1}$, months on the $x$ axis and depth (m) on the y axis. Dashed lines indicate the period of vertical mixing. 

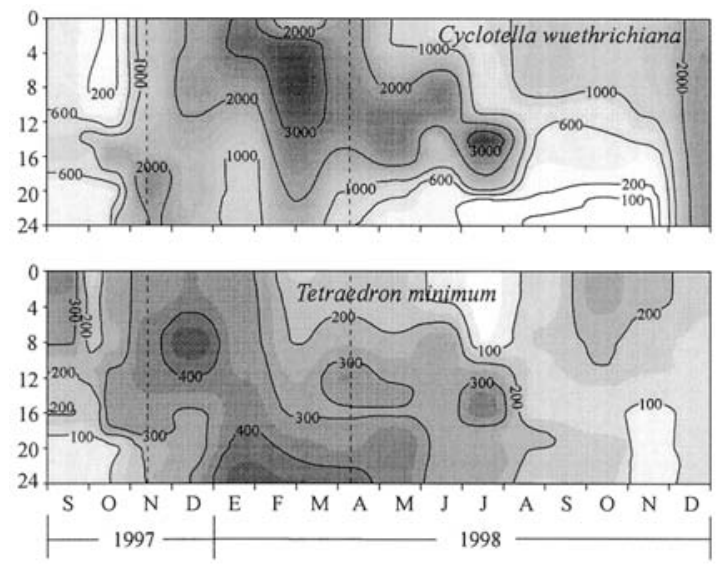

Figura 2 (continuación). Isolíneas de la densidad (cel ml-1) de las principales especies del nanofitoplancton durante el periodo de muestreo (meses, en abscisas) y en el perfil vertical (profundidad $\mathrm{m}$, en ordenadas). Las líneas discontinuas enmarcan el periodo de mezcla vertical de las aguas. (continuation) Timedepth distribution of main nanophytoplanktonic species. Density isopleths in cel $\mathrm{ml}^{-1}$, months on the $x$ axis and depth (m) on the $y$ axis. Dashed lines indicate the period of vertical mixing.

época de mezcla vertical (2500-3500 individuos/ml hasta los $8 \mathrm{~m}$ de profundidad) y al inicio de la estratificación. En el periodo invernal esta diatomea estaba bien representada a lo largo de toda la columna, y a medida que avanzaba la primavera su mayor abundancia se fue localizando en el metalimnion hasta principios de verano, para posteriormente disminuir notablemente desde mediados de verano hasta que recuperó su abundancia con la mezcla otoñal. Tetraedron minimum ((A.Br.) Hansg.) fue una de las especies más frecuentes de la laguna, contabilizándose en todas las muestras y profundidades. Su desarrollo más acusado se detectó en otoño de 1997, observándose un ligero aumento en la parte inferior de la columna de agua durante todo el periodo de mezcla que siguió. Esta especie se mantuvo más o menos abundante en el metalimnion, pero se redujo mucho en el epilimnion durante la estratificación. En otoño de 1998 no presentó un máximo acusado como en el año anterior, localizándose en las aguas superficiales. b) Especies del epilimnion o de aguas superficiales.

Ceratium hirundinella (O.F. Müller) se desarrolló preferentemente en el epilimnion durante el periodo de estratificación, alcanzando su máxima abundancia en la parte superior de la termoclina en septiembre de 1997. Pese a su escasa abundancia, debido a su gran tamaño es importante en términos de biovolúmenes. En la época de mezcla prácticamente desapareció, algo que ocurrió de forma más temprana en 1998 respecto a 1997. En los dos años estudiados varias especies de desmidiáceas pertenecientes al género Cosmarium experimentaron un notable desarrollo a finales del periodo de estratificación. Cosmarium laeve (Rabenhorst), y Cosmarium abbreviatum (Raciborski) presentaron máximos poblacionales en el epilimnion en dicha época, pero las densidades máximas, con valores semejantes, fueron alcanzadas alternativamente; en el caso de $C$. laeve esa mayor densidad poblacional se produjo en 1998 mientras que en C. abbreviatum se alcanzó en 1997. Cosmarium meneghinii (Bréb. in Ralfs) presentó también máximos poblacionales en la misma zona y época, pero más localizados en la superficie y con diferencias menos acusadas en los dos años.

\section{c) Especies del metalimnion-hipolimnion óxico o de profundidades intermedias.}

La mayor densidad poblacional de Cosmarium bioculatum (Bréb. ex Ralfs) se localizó en la parte más profunda del metalimnion a finales del periodo estival de 1997 y en la misma profundidad durante la estratificación de 1998, con densidades inferiores al resto de especies de Cosmarium. Sin embargo, fue muy destacable la acusada proliferación de $C$. cf. bioculatum desde mediados hasta el final de la estratificación de 1998, con un marcado máximo que fue descendiendo asociado al máximo del gradiente térmico, desde el entorno de los $8 \mathrm{~m}$ en julio hasta los $16 \mathrm{~m}$ en noviembre. Este aumento aportó los valores más elevados de densidad poblacional para el periodo estudiado, con un 
máximo de 32500 individuos $/ \mathrm{ml}$ a $14 \mathrm{~m}$, en septiembre de 1998. Es interesante destacar que C. cf. bioculatum no sólo proliferó intensamente, sino que esta especie se estableció ligeramente desplazada en el espacio y en el tiempo respecto a sus congenéricas. Chlorella $\mathrm{cf}$. vulgaris se detectó de forma bastante frecuente en el nanofitoplancton de la laguna, destacando su máxima abundancia sobre 16-18 m, en la parte superficial del hipolimnion, a finales del periodo de estratificación de 1997. Lagerheimia quadriseta ((Lemm.) G.H. Smith) alcanzó una elevada densidad poblacional prácticamente en toda la columna de agua durante el periodo de estudio de 1997, con un máximo metalimnético en noviembre. A finales de la época invernal 1997-98 inició un acusado descenso en su abundancia. A pesar de no alcanzar valores elevados de abundancia es interesante destacar la presencia de Synedra acus (Kützing) en la zona correspondiente al metalimnion a finales de la época de estratificación de 1997 y, en profundidades medias, durante el periodo de mezcla de aguas invernales. Tras la práctica desaparición de esta especie durante la primavera-verano, volvió a desarrollarse al final de la estratificación de 1998 a menor profundidad que el año anterior. Peridinium umbonatum (Stein) fue el dinoflagelado más frecuente y abundante en la laguna. Su máximo se observó a inicios del periodo de estratificación de 1998 en la parte superior del metalimnion, disminuyendo considerablemente en aguas más profundas. Durante los periodos de mezcla su presencia fue escasa en toda la columna de agua.

\section{d) Especies características de la oxiclina (durante la estratificación) o de aguas profundas.}

Las criptofíceas Cryptomonas erosa (Ehrenberg) y Cryptomonas phaseolus (Skuja) se encontraron en la zona de la oxiclina, especialmente la segunda. No obstante, durante la época de mezcla de aguas estas especies tuvieron un gran desarrollo en la parte superior de la columna, con un máximo más profundo de $C$. phaseolus res- pecto a C. erosa. Rhodomonas lacustris (Pascher et Ruttner) alcanzó una mayor densidad poblacional en la zona profunda, principalmente durante la época invernal 1997-98 y en la oxiclina durante la época estival de 1998. En cambio Rhodomonas minuta var. nannoplanctica (Skuja) presentó un máximo profundo a finales de la época de estratificación de 1997 y durante el periodo de mezcla prácticamente desapareció. Por tanto, las dos especies de Rhodomonas se excluyeron mutuamente: a finales de la estratificación de 1997 R. minuta dejó paso a una mayor presencia de $R$. lacustris en toda la columna de agua en la época de mezcla, mientras que durante la estratificación de 1998 R. lacustris se desarrolló más abundantemente en la oxiclina y $R$. minuta en el epilimnion y/o parte alta de la oxiclina al principio del periodo, cuando Rhodomonas lacustris era menos abundante (y posteriormente en diciembre se observó también una segregación espacial). Así mismo, se observa una segregación entre las poblaciones de los dos géneros de criptofíceas encontradas en la laguna (Cryptomonas y Rhodomonas), fenómeno más acentuado fuera del periodo de mezcla. Por su crecimiento restringido a capas profundas podemos destacar las crisofíceas, Chrysolykos planctonicus (Mack) y Bitrichia sp. que se desarrollaron respectivamente la primera a mediados-finales de verano y la segunda a principios de otoño. Con mucha menor abundancia se encontraron también varias especies de euglenófitos circunscritas a la interfase óxico-anóxica

La evolución estacional de estas especies (Figs. 2 y 3 ) se puede resumir como sigue. Durante el periodo invernal las poblaciones nanofitoplanctónicas de la Laguna del Tejo estaban dominadas por las diatomeas, principalmente Cyclotella wuethrichiana, siendo también relativamente abundantes las clorofíceas, especialmente Tetraedron minimum. Con el inicio de la estratificación a finales de primavera, la abundancia de diatomeas fue disminuyendo en la columna de agua, concentrándose en el metalimnion, pero mostrando un considerable descenso en la zona profunda. Esta disminución dio paso a un aumento de dinoflageladas (principalmente 


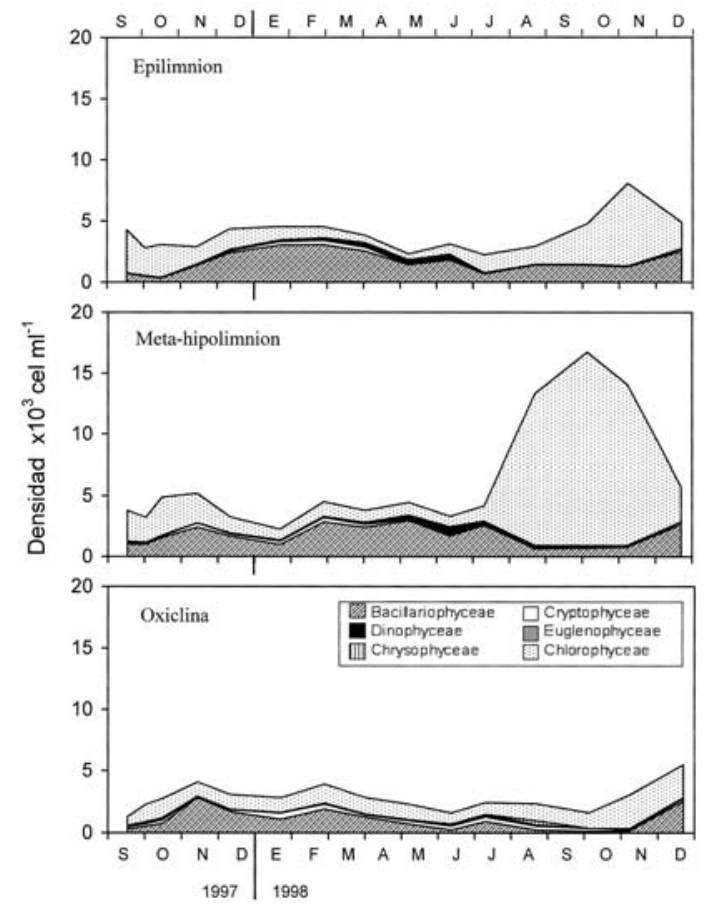

Figura 3. Variación temporal de la densidad de los distintos grupos algales (excluyendo las picocianobacterias) durante el periodo de estudio, diferenciando las distintas capas de la columna de agua durante la estratificación: epilimnion, metahipolimnion e interfase óxica-anóxica. Este último intervalo de profundidad fue el mismo para todo el año (desde $19 \mathrm{~m}$ hasta la profundidad de extinción de oxígeno), pero la divisoria entre los dos primeros varió según los meses del año (las muestras de $8,11,14$ y $16 \mathrm{~m}$ se fueron integrando sucesivamente en las del epilimnion; ver métodos). Durante el periodo de mezcla se mantuvieron los siguientes intervalos: 0-11 m, 12-18 m, 19$24 \mathrm{~m}$. Temporal density changes of algal groups (excluding picocyanobacteria) during the study period in the epilimnion, meta-hypolimnion and oxic-anoxic interface. The latter depth interval was always situated between $19 \mathrm{~m}$ depth and the oxygen extinction depth, while the limit between epilimnion and meta-hypolimnion varied depending on the month. Samples from $8,11,14$ and $16 \mathrm{~m}$ depth were succesively included in the epilimnion; see text for details. During mixing, depth intervals were: 0-11 $\mathrm{m}, 12-18 \mathrm{~m}$ and 19-24 $\mathrm{m}$.

Peridinium umbonatum), primero en superficie y después a profundidades mayores, en el metalimnion. Las criptofíceas, abundantes en toda la columna en invierno, se localizaron ya desde el inicio de la estratificación en la zona de la oxiclina, situación que se mantuvo durante todo el periodo estival. A finales de verano y en otoño de ambos años, las clorofíceas fueron el grupo
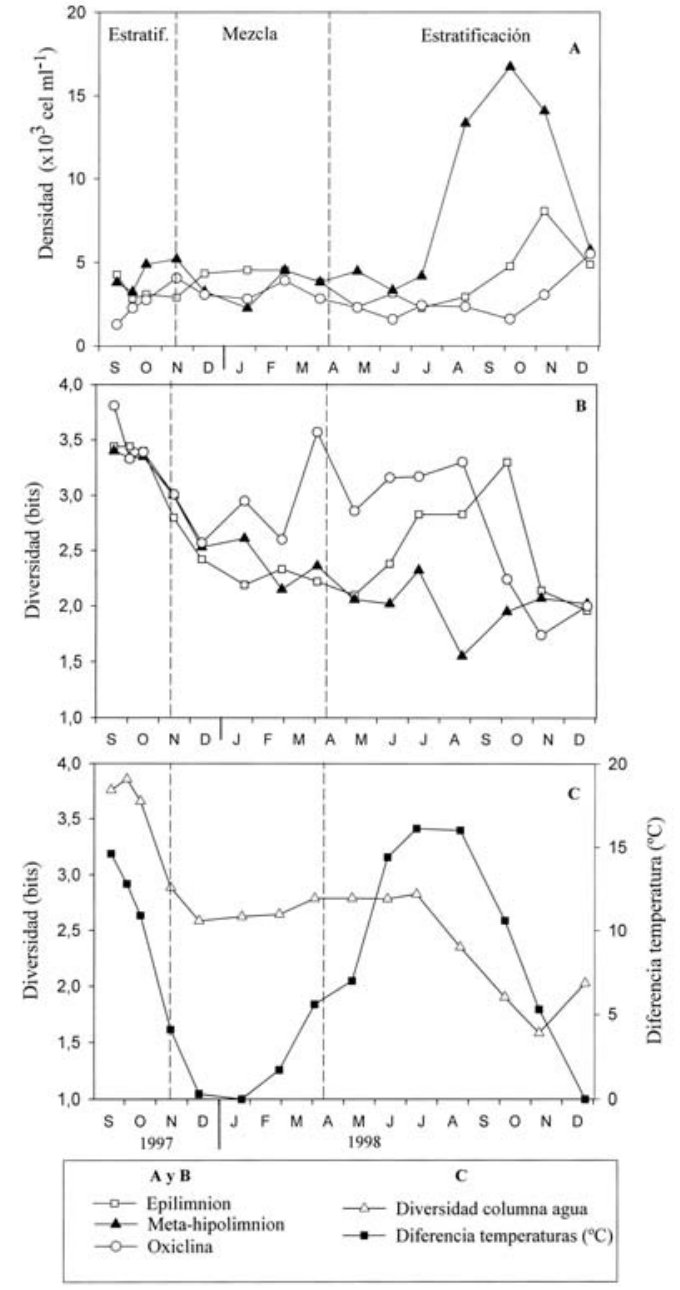

Figura 4. Distribución temporal de la densidad (A) y diversidad del nanofitoplancton (B) en los diferentes intervalos de profundidad. Variación temporal de la diversidad de la columna de agua y diferencia entre las temperaturas superficial y profunda (C). Las líneas verticales discontinuas enmarcan el periodo de mezcla de aguas. Intervalos de profundidad como en la figura 3. Temporal distribution of nanophytoplankton density $(\boldsymbol{A})$ and diversity $(\boldsymbol{B})$ for each depth interval. Temporal variation of the water column diversity and temperature differences between surface and bottom $(\boldsymbol{C})$. Dashed lines indicate the period of vertical mixing. Depth intervals as in figure 3.

nanofitoplánctónico dominante, constituyendo Cosmarium spp. más del 50\% de su población. A finales de verano-otoño de 1998 fue éste el género dominante de forma absoluta, con más del $50 \%$ de los individuos del nanofitoplancton en el metalimnion, en cambio en el otoño de 1997 no se produjo un crecimiento tan acusado 
de las especies de este género. Con el establecimiento de la mezcla vertical la dominancia se invirtió de nuevo a favor de las diatomeas. Este hecho ocurrió a finales de los dos años de estudio, aunque en 1998 el declive de las poblaciones de clorofíceas fue más drástico que en 1997.

\section{Abundancia, diversidad y heterogeneidad}

Por lo general, el número de individuos del nanofitoplancton por unidad de volumen no fue muy elevado (Fig. 4A), alcanzándose las mayores abundancias en el metalimnion en octubre de 1997 y de forma más acusada en septiembreoctubre de 1998. En el periodo invernal la máxima abundancia se localizó en aguas superficiales. La abundancia total del nanofitoplancton en la zona de la oxiclina fue siempre menor que en los estratos superiores. En la figura $4 \mathrm{~B}$ y C se representa la diversidad del nanofitoplancton, observándose que durante el periodo de estudio la oxiclina presentó los mayores valores de diversidad con una relativa menor fluctuación, disminuyendo respecto al resto de zonas únicamente a finales de la época estival de 1997 (Fig. 4B). Esto es debido a la escasa población nanofitoplanctónica, ya que a esta profundidad dominaba el picoplancton. También se contrastaron las diversidades de la columna de agua frente a la diferencia de temperaturas superfi- cial y profunda (19 m) de los perfiles verticales (Fig. 4C); la variación de ambos parámetros se espera que sea semejante y así fue durante los gradientes térmicos decrecientes (de septiembre a enero) cuando la diversidad de la columna de agua siguió el patrón de descenso esperado y durante el aumento del gradiente térmico al final de la estratificación de 1997 en que se alcanzaron los mayores valores de diversidad. Sin embargo durante la estratificación de 1998, debido a los crecimientos masivos de Cosmarium, el valor de diversidad de la columna de agua no aumentó, siendo la diversidad en el meta-hipolimnion inferior a la del epilimnion y coincidiendo los valores de menor diversidad con los de mayor abundancia (Fig. 4 A y B). Para relacionar la diversidad con el número de especies, en la Tabla 2 se detalla el número de especies encontradas distribuidas en los diferentes grupos nanofitoplanctónicos. El mayor número de especies en cualquiera de los meses considerados correspondió a las clorofíceas, aunque muchas de ellas no tuvieron importancia cuantitativa. El mayor valor total de especies distintas encontradas se obtuvo en agosto de 1998 y el menor número durante los meses invernales, observándose un descenso en el número de especies a principios de junio de 1998, después de un importante periodo de lluvias (Camacho et al., 2003).

Tabla 2. Número de especies encontradas pertenecientes a cada grupo nanofitoplanctónico y número total de especies en los diferentes meses del periodo estudiado. Number of species belonging to each nanophytoplanktonic group and total number of species found during sampling months (September 1997-December 1998).

\begin{tabular}{lcccccccccccccccccc}
\hline & \multicolumn{1}{c}{1997} & & & & & \multicolumn{1}{c}{1998} \\
\hline Grupos & $\mathrm{S}$ & $\mathrm{O}$ & $\mathrm{O}$ & $\mathrm{N}$ & $\mathrm{D}$ & $\mathrm{E}$ & $\mathrm{F}$ & $\mathrm{M}$ & $\mathrm{My}$ & $\mathrm{Jn}$ & $\mathrm{Jl}$ & $\mathrm{A}$ & $\mathrm{S}$ & $\mathrm{N}$ & $\mathrm{D}$ \\
\hline Bacillariophyceae & 5 & 6 & 7 & 5 & 7 & 5 & 5 & 5 & 7 & 5 & 5 & 5 & 5 & 6 & 4 \\
Cryptophyceae & 3 & 5 & 4 & 4 & 7 & 7 & 6 & 6 & 7 & 7 & 5 & 9 & 5 & 6 & 8 \\
Dinophyceae & 5 & 6 & 5 & 2 & 3 & 2 & 2 & 4 & 3 & 3 & 6 & 4 & 3 & 3 & 3 \\
Euglenophyceae & 2 & 2 & 3 & 0 & 1 & 1 & 0 & 1 & 1 & 0 & 2 & 1 & 1 & 0 & 0 \\
Chrysophyceae & 3 & 2 & 3 & 0 & 1 & 0 & 0 & 0 & 0 & 0 & 0 & 1 & 3 & 4 & 2 \\
Cianophyceae & 1 & 1 & 1 & 1 & 1 & 1 & 0 & 1 & 0 & 0 & 1 & 1 & 0 & 0 & 0 \\
Chlorophyceae & 20 & 25 & 19 & 20 & 20 & 23 & 26 & 27 & 36 & 24 & 30 & 35 & 24 & 21 & 20 \\
\hline Total especies & 39 & 47 & 42 & 32 & 40 & 39 & 39 & 44 & 54 & 39 & 49 & 56 & 41 & 40 & 37 \\
\hline
\end{tabular}




\section{Asociaciones}

Los resultados del Análisis de Componentes Principales (PCA) ponen de manifiesto aquellos factores que determinaron los cambios en la población nanofitoplanctónica a lo largo del perfil vertical y del ciclo limnológico anual (Figs. 5 y 6). Los dos primeros factores principales obtenidos en el PCA explicaron de forma conjunta aproximadamente el $60 \%$ de la varianza total de los datos. Las muestras representadas por los valores de estos factores se presentan separadamente en dos gráficos (Fig. 5, A y B) dividiendo en dos zonas la columna de agua (0-13 m y
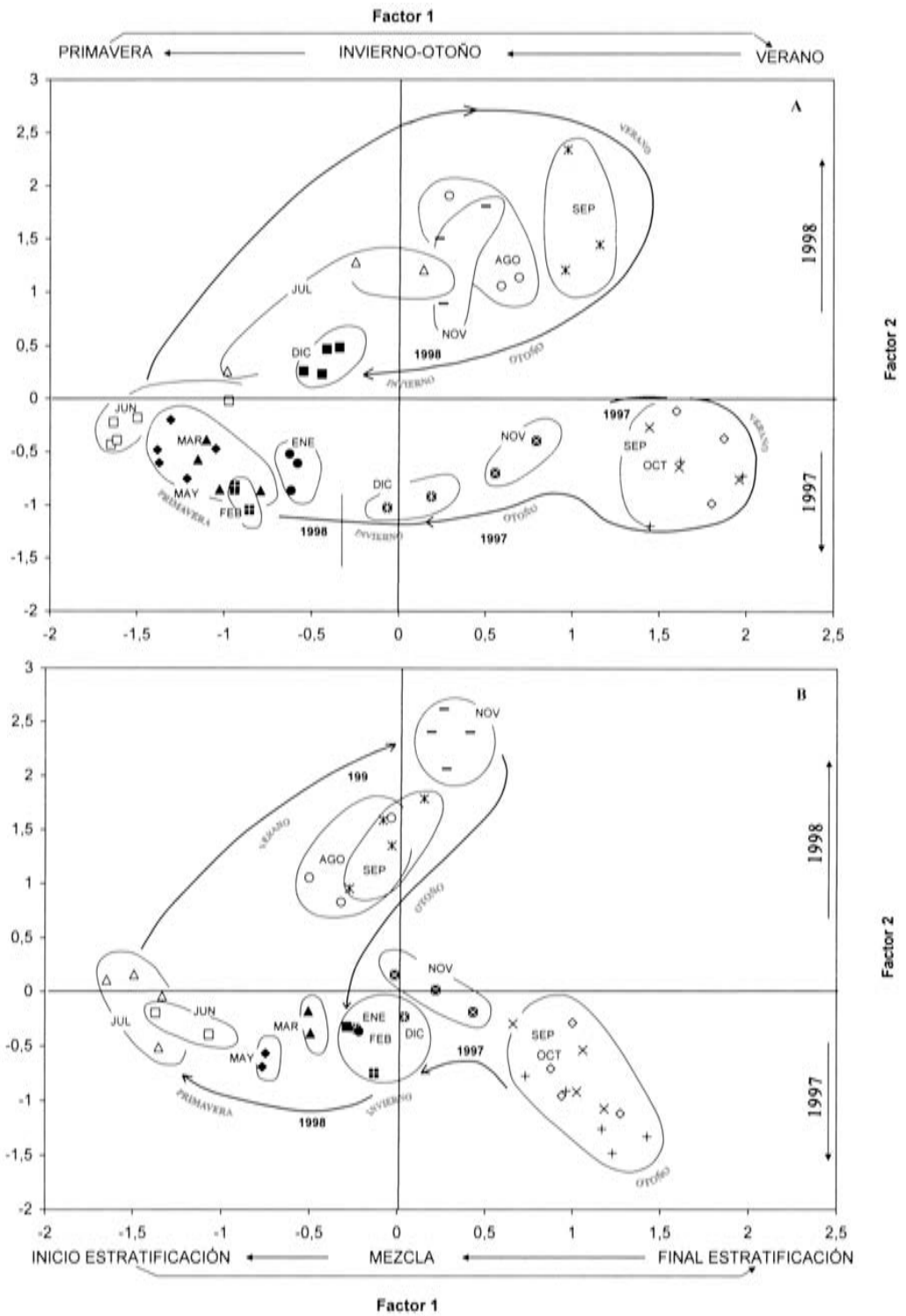

Figura 5. Situación de las muestras en el espacio definido por los dos primeros factores resultantes del PCA. A: aguas superficiales $(0-13 \mathrm{~m})$ y B: aguas profundas (14-22/24 m). Los distintos símbolos corresponden a los meses muestreados que se indican por sus siglas en la correspondiente agrupación. Position of the samples in the space defined by the first two factors from the PCA. A: surface waters $(0-13 \mathrm{~m})$ and $\boldsymbol{B}$ : deep waters $(14-22 / 24 \mathrm{~m})$. Symbols represent the different sampling months, which are abbreviated in the corresponding clusters. 
14-22 m, ó $24 \mathrm{~m}$ ), porque la variabilidad en las muestras de superficie y profundidad es muy diferente. Como profundidad divisoria se han escogido los $14 \mathrm{~m}$, que es la profundidad donde, en la mayoría de los meses después del máximo metalimnético, empieza a disminuir el oxígeno. Las muestras se ubican siguiendo una secuencia respecto a los meses del año, mostrando el primer factor la variación entre el inicio y el final de la estratificación y el segundo la diferencia interanual entre los meses del final de la estratificación (septiembre-noviembre) de 1997 y de 1998. Los valores correspondientes a la época de mezcla de aguas quedan juntos y cercanos al valor 0 . Esto se hace más evidente en las muestras de profundidad (Fig. 5B) concentrándose las pertenecientes al periodo de mezcla en las proximidades del cruce de ambos ejes. Las muestras de profundidad son siempre mucho menos variables que las de la parte superficial: (1) las del final del periodo de estratificación quedan mucho más centradas respecto al primer factor principal y (2) las del inicio y establecimiento de la estratificación con mucha menor variabilidad respecto a los dos factores (junio y julio quedan juntos en profundidad (Fig. 5B), en cambio las muestras de la parte superficial del mes de julio se diferencian mucho (Fig. 5A)).

Las correlaciones de las especies nanofitoplanctónicas con los factores principales se representan en la figura 6 y agrupan aquellas especies que requerían condiciones semejantes para su desarrollo. En primer lugar, las especies de presencia más frecuente, Cyclotella wuethrichiana y Tetraedron minimum, se sitúan con correlaciones negativas para los dos factores, con valores más bajos para el primer factor, situándose las especies de las distintas capas del meta e hipolimnion estival con correlaciones negativas más altas del primer factor (variando con el segundo factor). En la parte más positiva de este primer factor se encuentran las especies de Cosmarium de presencia superficial al final de los periodos de estratificación. En la parte más positiva del segundo factor se sitúan las especies del hipolimnion de finales de la estratificación de 1998 (septiem- bre-noviembre). Por otro lado, en la parte negativa del segundo factor y positiva del primero se sitúan las especies abundantes en el mismo periodo que acabamos de citar pero del año 1997. Por lo demás, los resultados del análisis ponen de manifiesto la contraposición entre algunas especies congenéricas. Son de destacar las siguientes contraposiciones:

1) Cryptomonas erosa y Rhodomonas minuta var. nannoplanctica se sitúan en posiciones opuestas a Cryptomonas phaseolus y Rhodomonas lacustris. Las primeras se desarrollaron abundantemente durante el final de la estratificación de 1997 en profundidad y en todo el perfil en la primera parte de la época invernal, pero el resto del periodo de estudio su abundancia decreció, mientras que las segundas se desarrollaron especialmente al final del invierno en todo el perfil y en su parte profunda durante la estratificación de 1998, especialmente los primeros meses. Además, en las fechas en que coincidían, el máximo de abundancia de las segundas especies siempre se situaba más profundamente, si bien debe considerarse que las especies del género Rhodomonas estaban por lo general más próximas a las capas profundas del hipolimnion que las de Cryptomonas.

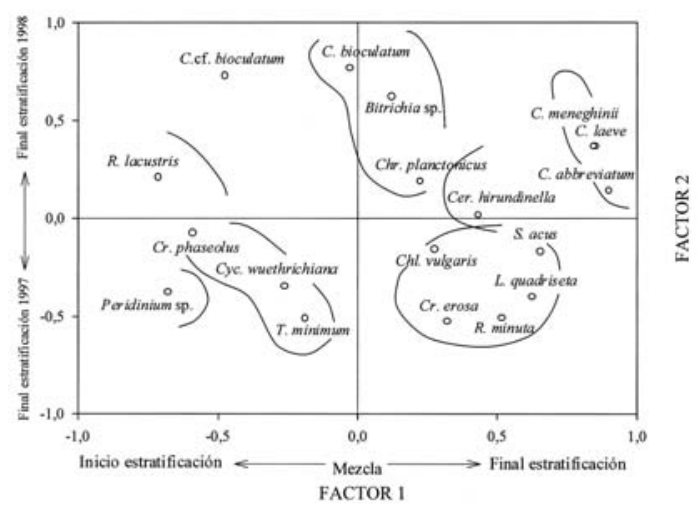

Figura 6. Situación de las principales especies nanofitoplanctónicas en el espacio definido por los dos primeros factores resultantes del PCA. Las especies se han agrupado según su distribución espacio-temporal. Position of the main nanophytoplanktonic species in the space defined by the first two principal factors from the PCA. Species are grouped according to their spatio-temporal distribution. 
2) Algunas especies de Cosmarium se separan por sus coeficientes de correlación porque fueron alternativamente abundantes en uno u otro año al final de la estratificación. C. cf. bioculatum presentó las mayores diferencias con respecto a las otras especies del género, al constituirse como la especie dominante en el metalimnion del final de la estratificación de 1998 y C. bioculatum también fue más abundante en 1998 respecto a la misma época que el año anterior. Por otra parte, C. meneghinii, C. laeve y C. abbreviatum presentaron máximos de densidad poblacional en superficie al final de la estratificación en ambos años, siendo más importante en 1997 para C. abbreviatum.

3) Las dos especies principales de dinoflageladas se separan también en el espacio de los factores principales: P. umbonatum se localizó principalmente al inicio de la estratificación en el metalimnion y $C$. hirundinella en el epilimnion durante la época estival.

\section{DISCUSIÓN}

La marcada estratificación estival de la laguna cárstica del Tejo influyó de manera notable en la distribución de las poblaciones fitoplanctónicas. La gran profundidad relativa de esta laguna, su morfología circular y su ubicación hundida en la dolina, la protegen de los eventos metereológicos y favorecen el establecimiento de una estratificación térmica vertical y la estructuración de los gradientes de oxígeno, produciéndose en la zona profunda una interfase óxico-anóxica. Los productores primarios planctónicos se organizan en el perfil vertical en dos máximos de población, uno debido al nanoplancton eucariota situado en el metalimnion (Fig. 1D) y otro a las picocianobacterias en la interfase óxico-anóxica, que se evidencia por el máximo profundo de clorofila $a$ (Fig. 1C). La gran importancia del picoplancton se pone de manifiesto al no coincidir la distribución de clorofila $a$ con la del total de algas del nanoplancton. Estas elevadas concentraciones de clorofila $a$ en la interfase óxicoanóxica son debidas al desarrollo de densas poblaciones de picoplancton fotoautotrófico tipo Synechococcus (Camacho et al., 2003). Estas cianobacterias proliferan considerablemente por la mayor disponibilidad de nutrientes en dicha interfase y gracias a pigmentos accesorios como la ficoeritrina que permiten el aprovechamiento óptimo de la escasa luz que alcanza dichas profundidades. Por tanto, a pesar de que el presente estudio se centra en las poblaciones nanofitoplanctónicas debemos constatar la importancia del picoplancton procariota en la estructura trófica del lago. Estos máximos profundos de clorofila también se han detectado en otras lagunas cercanas, como la Laguna de La Cruz (Vicente \& Miracle, 1988), el Lagunillo del Tejo (Vicente \& Miracle, 1984) y la Laguna de Arcas-II (Camacho et al., 2000).

Respecto al nanofitoplancton, la baja densidad de individuos que por lo general se observa puede relacionarse con la baja disponibilidad de nutrientes inorgánicos. En las aguas ricas en calcio, como en la Laguna del Tejo, es característica la rápida precipitación del fósforo en forma de apatito, especialmente en aguas de $\mathrm{pH}$ alcalino. Al inicio de la estratificación, el fósforo constituyó el factor limitante para el desarrollo de las especies algales, tal y como se ha citado para los lagos de regiones templadas (Schindler, 1978). Sin embargo, a finales del periodo de estratificación de 1997, el nitrógeno controló el desarrollo algal en el epi-metalimnion, según los bioensayos realizados adicionalmente a la toma de muestras en este periodo (Camacho et al., 2003). Este cambio en el control por nutrientes sobre los productores primarios ha sido descrito para otros lagos de zonas templadas (Lehman \& Sandgen, 1978; Planas, 1990).

La sucesión estacional del nanofitoplancton de la Laguna del Tejo es similar a la descrita en otros lagos cársticos, pero se diferencia principalmente en el excepcional crecimiento de las especies de Cosmarium al final del periodo de estratificación. Así, la presencia de importantes poblaciones de Cyclotella prácticamente todo el año, un mayor número dentro del grupo de las clorofíceas, y la estructuración de la comunidad nanofitoplanctónica con un reducido número de 
especies presentando elevadas abundancias, se ha descrito para otros lagos de la Península Ibérica de naturaleza cárstica, como los lagos de Banyoles (Planas, 1973), Montcortés (Camps et al., 1976), Estanya (Ávila et al., 1984), laguna del Vilà (García de Emiliani, 1973) y la cercana laguna de La Cruz (Rojo y Miracle, 1987; Dasí y Miracle, 1991, Miracle et al., 1998). En la mayoría de estos lagos también se han constatado crecimientos explosivos de clorofíceas, pero nunca de Cosmarium, así como la importancia del establecimiento de poblaciones bien definidas en la interfase óxico-anóxica, especialmente de los grupos de criptofíceas, crisofíceas y euglenofíceas. En concreto $C$. phaseolus se cita como característica de la oxiclina en la mayoría de las lagos antedichos y en las lagunas del Cisó (Gasol et al., 1990) y de Arcas (Camacho et al., 2001).

La sucesión en la Laguna del Tejo se puede resumir como sigue: (1) En superficie viene determinada por el descenso en la disponibilidad de nutrientes (y entre ellos el $\mathrm{Si}$ ): Cyclotella-Peridinium - Ceratium. (2) A finales de verano, debido a perturbaciones físicas (viento, disminución de la temperatura en el litoral) que conducen a la profundización de la termoclina y generan también corrientes litoralcentro que, en esta pequeña laguna, facilitan el transporte horizontal de nutrientes y algas o diásporas por el metalimnion superficial, de manera que la sucesión retrocede en esta capa y se produce un crecimiento de Cosmarium. Reynolds en una revisión (Reynolds \& Reynolds, 1985) describe también estos retrocesos en la sucesión por las perturbaciones físicas de finales de verano y presenta datos de lagos mesotróficos del Reino Unido donde estas regresiones llevan a un aumento de especies de desmidiáceas (Cosmarium entre ellas).

Es destacable la gran densidad poblacional del grupo de las desmidiáceas a finales del periodo de estratificación de 1998, cuando se produce en el metalimnion desde mediados de agosto un intenso crecimiento de C. cf. bioculatum que conduce a un aumento de su población en más de un orden de magnitud. Esta prolifera- ción denota unas condiciones en el lago que no ocurrieron en su metalimnion el año anterior, por ejemplo la incidencia de vientos más intensos y mayores cambios de temperatura dia/noche, que podrían aumentar la circulación de agua desde el litoral al centro, vía metalimnion superficial, debido al gradiente térmico más agudo que se forma cuando la termoclina se profundiza. Además, el agotamiento de nutrientes tras la época estival pudo inducir una intensa competencia por la adquisición del recurso justo en esta estrecha capa de gradiente acusado, que condujo a la proliferación de una determinada especie. Es destacable como $C$. cf. bioculatum va situando su máximo sucesivamente más profundo, siempre unos metros por debajo del máximo metalimnético de oxígeno, dominando absolutamente en dicha zona hasta que la mezcla vertical se completa.

La bibliografía refiere que en lagos de aguas alcalinas el desarrollo de desmidáceas no está favorecido y también que no es frecuente que sea un grupo dominante en el plancton (Hutchinson, 1967). Sin embargo esta misma referencia, extraordinaria obra de compilación del conocimiento del fitoplancton hasta aquella fecha, ya recoge citas antiguas de C. bioculatum var. minutum produciendo máximos poblacionales asociados a una cianofícea chroococal en las montañas de Java (Ruttner, 1952) y de $C$. bioculatum como dominante junto con cianofíceas en lagos finlandeses eutróficos (Järnefelt, 1956). Además, estudios recientes demuestran su proliferación en este tipo de aguas en condiciones de limitación de fósforo inorgánico debido a la alta actividad de su enzima fosfatasa alcalina (Spijkerman \& Coesel, 1998). Es posible la existencia de una asociación con máximos de abundancia de Cosmarium, acompañado de una cianofícea (en nuestro caso Synechococcus) con su máximo situado en una posición más profunda.

La presencia prácticamente constante y normalmente dominante de $C$. wuethrichiana, el desarrollo superficial de dinofíceas durante la estratificación y la adaptación de las especies a una baja disponibilidad de nutrientes indican la 
naturaleza oligotrófica de las aguas superficiales de la Laguna del Tejo, pero el desarrollo de los Cosmarium en algunos años denota su condición de mesotrofia. Por ello en aguas profundas se presenta una zona en la que domina la descomposición donde se desarrollan Cryptophyceae y Chrysophyceae. Es destacable la proliferación de crisofíceas en la interfase óxico-anóxica al final de la estratificación de 1998, relacionando su presencia con la comentada existencia de unas condiciones distintas en la laguna respecto al año anterior. En la cercana Laguna de La Cruz se observó que, aunque este grupo fue poco representativo, también se localizó preferentemente en la oxiclina (Dasí y Miracle, 1991).

Los máximos profundos de $T$. minimum y $C$. wuethrichiana se podrían deber en ocasiones a sedimentación pasiva y retención por diferencias de densidad en el agua y a una mayor resistencia a la degradación de los individuos sedimentados. La composición de sílice de los frústulos de las diatomeas y la pared celular altamente resistente de T. minimum compuesta por biopolímeros alifáticos no hidrolizables (Blokker et al., 1998), permiten su preservación en las aguas profundas y en los sedimentos.

Esta laguna pertenece al grupo de lagos en los que, durante la estratificación, la luz penetra hasta la interfase óxico-anóxica, donde se produce el reciclaje de los nutrientes que promueven la aparición de densas poblaciones algales en la oxiclina (Reynolds, 1992). En esta zona fronteriza proliferan intensamente las cianobacterias tipo Synechococcus y las criptofíceas, con un uso diferencial de los recursos. Experiencias realizadas con criptofíceas en la Laguna del Cisó (Gasol et al., 1990) indican su mejor adaptación a bajas intensidades luminosas al avanzar la estratificación.

La distribución y desarrollo de las especies algales de la Laguna del Tejo durante el periodo de estudio muestran que se da un patrón distinto de los valores del índice de diversidad en un año con abundantes precipitaciones primaverales como el 1998, en comparación al año anterior más seco. Los valores de diversidad obtenidos siguen un patrón de comportamiento espacial y temporal que aumenta con el gradiente térmico, es decir con la estratificación, en 1997, pero no en 1998, en el que la diversidad disminuye por la proliferación masiva de C. cf. bioculatum, cuando se da el mayor gradiente de temperaturas (Fig. 4C).

El PCA indica que la comunidad algal se desarrolla preferentemente de acuerdo a los factores que determinan las diferencias existentes entre la época de estratificación y la época de mezcla, mostrando que dichos factores difieren para el periodo de estratificación en ambos años. Éste confirma además la comentada estabilidad de la zona de agua profunda que presenta una menor variación que la zona superficial durante la sucesión estacional. El presente trabajo incide en la importancia de condicionantes abióticos en la composición y estructuración de la comunidad nanofitoplanctónica a lo largo del tiempo. La sucesión inducida por efecto de la turbulencia en sistemas estratificados es referida por Reynolds $(1980,1984)$, señalando la disponibilidad de nutrientes y la estabilidad de la columna como los factores más importantes para los acontecimientos de la sucesión. Además, los cambios en la turbulencia fueron asociados a la formación de crecimientos algales explosivos, normalmente de cianofíceas (Reynolds \& Walsby, 1975). Sin embargo, estudios más recientes (Reynolds, 1997) señalan la influencia de los cambios temporales sobre la estructura de la comunidad no como motores de la sucesión sino promoviendo un "ensamblado" entre especies. En conclusión, el cambio detectado en lo que se refiere a la comunidad nanofitoplanctónica a lo largo del periodo de estudio en la Laguna del Tejo permite definir dicha comunidad por su entidad heterogénea, siendo los factores de estacionalidad y verticalidad los que en principio la condicionan.

\section{AGRADECIMIENTOS}

Este trabajo fue financiado por el proyecto CLI961103-CO2-01 (CICYT) dirigido por M.R.M.

\section{BIBLIOGRAFÍA}

ANAGNOSTIDIS, K. \& J. KOMÁREK. 1988. Modern approach to the classification system of 
cyanophytes. 3-Oscillatoriales. Arch. Hydrobiol. Suppl. 80, (Algological Studies 50-53): 327-472.

ANTON, A. \& H. C. DUTHIE. 1981. Use of cluster analysis in the systematics of the algal genus Cryptomonas. Can. J. Bot., 59: 992-1002.

APHA-AWWA-WEF. 1992. Standard methods for the examination of water and wastewater. $18^{\text {th }}$ edition. American Public Health Association. Washington D.C.

ÁvilA, A., J. L. BURREL, A. DOMINGO, E. FERNÁNDEZ, J. GODALL \& J. M. LLOPART. 1984. Limnología del Lago Grande de Estanya (Huesca). Oecologia aquatica, 7: 3-24.

BLOKKER, P., S. SCHOUTEN, H. VAN DEN ENDE, J. W. DE LEEUW, P. G. HATCHER \& J. S. S. DAMSTE. 1998. Chemical structure of algaeans from the fresh water algae Tetraedron minimum, Scenedesmus communis and Pediastrum boryanum. Organic Geochemistry, 29: 1453-1468.

CAMACHO, A., E. VICENTE \& M. R. MIRACLE. 2000. Ecology of a deep-living Oscillatoria (= Planktothrix) population in the sulphide-rich waters of a Spanish karstic lake. Arch. Hydrobiol., 148: 333-355.

CAMACHO, A., E. VICENTE \& M. R. MIRACLE. 2001. Ecology of Cryptomonas at the chemocline of a karstic sulfate-rich lake. Mar. Freshwater Res., 52: 805-815.

CAMACHO, A., W. A. WURTSBAUGH, M. R. MIRACLE, X. ARMENGOL \& E. VICENTE. Nitrogen limitation for phytoplankton at the end of the stratification period in a karst lake with a deep chlorophyll layer: a nutrient enrichment bioassay approach. (2003). J. Plankton Res., 25: 397-404

CAMPS, J., I. GONZALVO, J. GÜELL, P. LÓPEZ, A. TEJERO, X. TOLDRÁ, F. VALLESPINÓS \& M. VICENS. 1976. El lago de Montcortès: descripción de un ciclo anual. Oecologia aquatica, 2: 99-110.

DASÍ, M. J. Y M. R. MIRACLE. 1991. Distribución vertical y variación estacional del fitoplancton de una laguna cárstica meromíctica, la Laguna de La Cruz (Cuenca, España). Limnetica, 7: 37-59.

FÖRSTER, K. 1982. Das Phytoplankton des Süsswassers. Systematik und Biologie. Conjugatophyceae: Zygnematales und Desmidiales (excl. Zygnemataceae). Teil 8. In: Die Binnengewässer. Einzeldarstellungen aus der Limnologie und ihren Nachbargebieten. Elster,
H.J. and W. Ohle (eds.). E. Schweizerbart'sche Verlag. Stuttgart.

GARCÍA DE EMILIANI, M. O. 1973. Fitoplancton de la Laguna del Vilà (Gerona, España). Oecologia aquatica, 1: 107-155.

GASOL, J. M., J. MAS, C. PEDRÓS-ALIÓ Y R. GUERRERO. 1990. Ecología microbiana y limnología en la Laguna Cisó: 1976-1989. Scientia gerundensis, 16: 155-178.

GERMAIN, H. 1981. Flore Des Diatomées (eaux douces et saumâtres). E. Boubée (Societé Nouvelle des Éditions Boubée), Paris.

GOLTERMAN, H. L., R. S. CLYMO \& M. A. M. OHNSTAD. 1978. Methods for physical and chemical analysis of fresh waters. IBP Handbook no. 8, Blackwell Sci. Pub., Oxford, UK.

HUBER-PESTALOZZI, G. 1955. Das Phytoplankton des Süsswassers. Systematik und Biologie. Euglenophyceen. Teil 4. In: Die Binnengewässer. Einzeldarstellungen aus der Limnologie und ihren Nachbargebieten. Thienemann, A. (ed.). E. Schweizerbart'sche Verlag. Stuttgart.

HUBER-PESTALOZZI, G. 1968. Das Phytoplankton des Süsswassers. Systematik und Biologie. Cryptophyceae, Chloromonadophyceae, Dinophyceae. Teil 3. In: Die Binnengewässer. Einzeldarstellungen aus der Limnologie und ihren Nachbargebieten. Thienemann, A. (ed.). E. Schweizerbart'sche Verlag. Stuttgart.

HUTCHINSON, G. E. 1967. A Treatise on Limnology. Vol. 2: Introduction to Lake Biology and Limnoplankton. New York: Wiley.

JÄRNEFELT, H. 1956. Zur limnologie einigen Geuvässer Finnlands XVI. Mit besonderer Berücksichtigung des Planktons. Anls Zool. Soc. Vanamo, 17 (7): 201 pp.

JEFFREY, S. W. \& G. F. HUMPHREY. 1975. New spectrophotometric equations for determining chlorophylls $a, b, c_{1}$ and $c_{2}$ in higher plants, algae and natural phytoplankton. Biochem. Physiol. Pfl.,167: 191-194.

KOMÁREK, J. \& B. FOTT. 1983. Das Phytoplankton des Süsswassers. Systematik und Biologie. Chlorophyceae (Grünalgen). Ordung: Chlorococcales. Teil 7. In: Die Binnengewässer. Einzeldarstellungen aus der Limnologie und ihren Nachbargebieten. Elster, H.J. and W. Ohle (eds.). E. Schweizerbart'sche Verlag. Stuttgart.

KRAMMER, K. \& H. LARGE-BERTALOT. 19861988-1991a-1991b. Teil 2: Bacillariophyceae. (2/1): 
Naviculaceae; (2/2): Bacillariaceae, Epithemiaceae, Surirellaceae; (2/3): Centrales, Fragilariaceae, Eunotiaceae; and (2/4): Achnanthaceae. In: Süsswasserflora von Mitteleuropa. Ettl, H., J. Gerloff, H. Heynig and D. Mollenhauer (eds.). Gustav Fischer Verlag. Stuttgart, Jena.

LEHMAN, J. T. \& C. D. SANDGEN. 1978. Documenting a seasonal change from phosphorus to nitrogen limitation in a small temperate lake, and its impact on the population dynamics of Asterionella. Verh. Internat. Verein. Limnol., 20: 375-380.

MIRACLE, M. R., M. J. DASÍ \& E. VICENTE. 1998. Forced phytoplankton vertical migration due to lake water "whiting". Verh. Internat. Verein. Limnol., 26: 1749-1754.

PLANAS, D. 1973. Composición, ciclo y productividad del fitoplancton del lago de Banyoles. Oecologia Aquatica, 1: 3-106.

PLANAS, D. 1990. Factores de control de la distribución espacial y temporal de la producción primaria del fitoplancton del Lago de Banyoles. Scientia Gerundensis, 16/2: 193-204.

POPOVSKÝ, J. \& L. A. PFIESTER. 1990. Teil 6: Dinophyceae (Dinoflagellida). In: Süsswasserflora von Mitteleuropa. Ettl, H., J. Gerloff, H. Heynig and D. Mollenhauer (eds.)., Gustav Fischer Verlag. Stuttgart, Jena.

REYNOLDS, C. S. 1980. Phytoplankton assemblages and their periodicity in stratifying lake systems. Holarctic Ecol., 3: 141-159.

REYNOLDS, C. S. 1984. The ecology of freshwater phytoplankton. Cambridge University Press, Cambridge.

REYNOLDS, C. S. 1992. Dynamics, selection and composition of phytoplankton in relation to vertical structure in lakes. Arch. Hydrobiol. Beih. Ergebn. Limnol., 35: 12-31.

REYNOLDS, C. S. 1997. Vegetation processes in the pelagic: a model for ecosystem theory. In: Excellence in Ecology series. O. Kinne, Ed. Ecology Institute, Oldendorf/Luhe. Germany.

REYNOLDS, C. S. \& A. E. WALSBY. 1975. Waterblooms. Biol. Rev., 50: 437-481.
REYNOLDS, C. S. \& J. B. REYNOLDS. 1985. The atipycal seasonality of phytoplankton in Crose Mere, 1972: An independent test of the hypothesis that variability in the physical environment regulates community dynamics and structure. $B r$. phycol. J., 20: 227-242.

ROJO, C. Y M. R. MIRACLE. 1987. Poblaciones fitoplanctónicas de la Laguna de La Cruz, una laguna cárstica meromíctica. Act. VI Simp. Nac. Bot. Cript. Granada (Spain): 119-135.

RUTTNER, F. 1952. Planktonstudien der Deutschen Limnologischen Sundan Expedition. Arch. Hydrobiol., Suppl., 21: 1-274.

SCHINDLER, D. W. 1978. Factors regulating phytoplankton production and standing crop in the world's freshwaters. Limnol. Oceanogr., 23: 478-486.

SHANNON, C. E. \& W. WEAVER. 1963. The mathematical theory of communication. Univ. Illinois Press, Urbana.

SHOAF, W. T. \& B. W. LIUM. 1976. Improved extraction of chlorophyll $a$ and $b$ from algae using dimethyl sulphoxide. Limnol. Oceanogr., 21: 926928.

SPIJKERMAN, E. \& P. F. M. COESEL. 1998. Alkaline phosphatase activity in two planktonic desmid species and the possible role of an extracellular envelope. Freshwater Biol., 39: 503-513.

STARMACH, K. 1985. Teil 1: Chrysophyceae und Haptophyceae. In: Süsswasserflora von Mitteleuropa. Ettl, H., J. Gerloff, H. Heynig and D. Mollenhauer (eds.)., Gustav Fischer Verlag. Stuttgart, Jena.

UTERMÖHL, H. 1958. Zur Vervollkommung der quantitativen Phytoplankton-Methodik. Mitt. Int. Verein. Theor. Angew. Limnol., 9: 1-38.

VICENTE, E. \& M. R. MIRACLE. 1984. Distribution of photosinthetic organisms in a temporal stratified karstic pond near Cuenca, Spain. Verh. Internat. Verein. Limnol., 22: 1704-1710.

VICENTE, E. \& M. R. MIRACLE. 1988. Physicochemical and microbial stratification in a meromictic karstic lake of Spain. Verh. Internat. Verein. Limnol., 23: 522-529. 